Ww

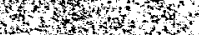

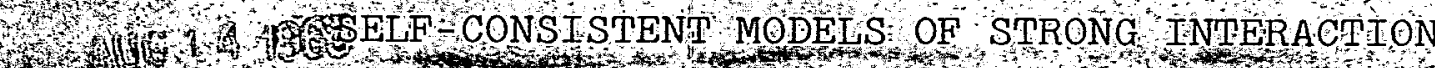

3x+m

(2) 3 , th

4

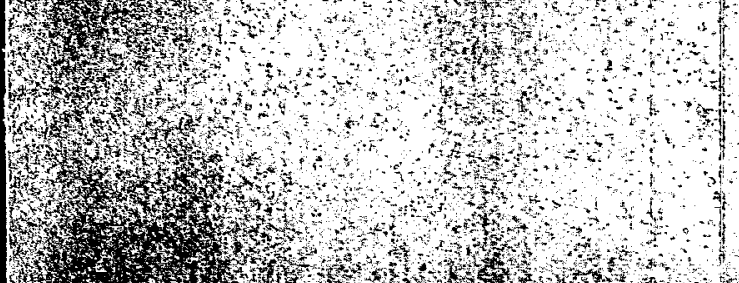

(t) WTTH CHXRAT, SYMMETRY

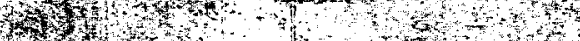

Wól chío Nambu and Pedro Pascual
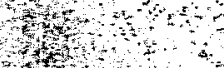

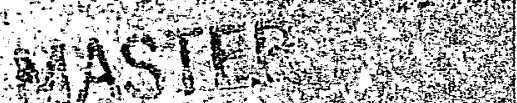

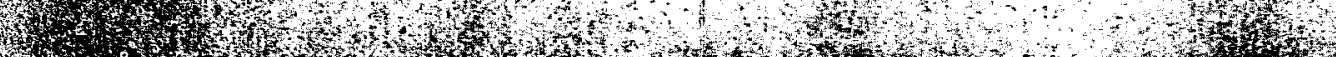

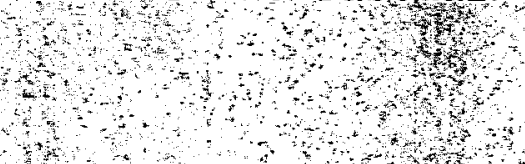

and

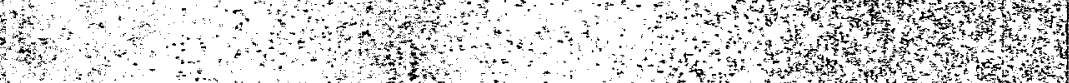

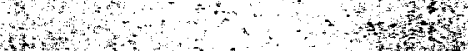

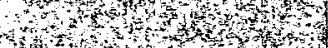

A

W

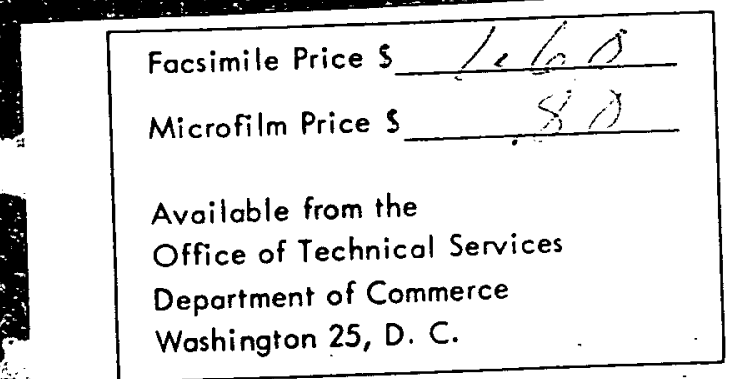

M

Wos
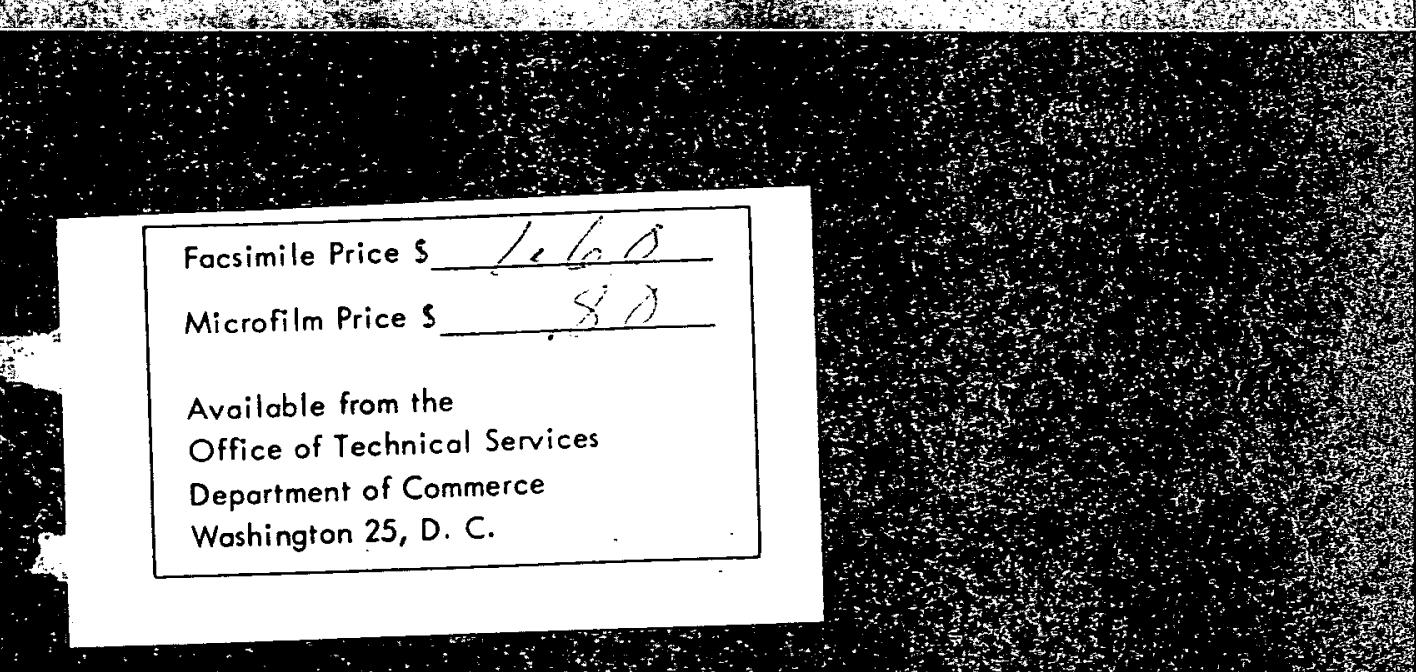


\section{DISCLAIMER}

This report was prepared as an account of work sponsored by an agency of the United States Government. Neither the United States Government nor any agency Thereof, nor any of their employees, makes any warranty, express or implied, or assumes any legal liability or responsibility for the accuracy, completeness, or usefulness of any information, apparatus, product, or process disclosed, or represents that its use would not infringe privately owned rights. Reference herein to any specific commercial product, process, or service by trade name, trademark, manufacturer, or otherwise does not necessarily constitute or imply its endorsement, recommendation, or favoring by the United States Government or any agency thereof. The views and opinions of authors expressed herein do not necessarily state or reflect those of the United States Government or any agency thereof. 


\section{DISCLAIMER}

Portions of this document may be illegible in electronic image products. Images are produced from the best available original document. 


\section{WITH CHIRAL SYMMETRY \\ Yoichiro Nambu \\ and \\ Pedro Pascual}

SELF-CONSISTENT MODELS OF STRONG INTERACTION

The Enrico Fermi Institute for Nuclear Studies

and the Department of Physics

The University of Chicago, Chicago, Illinois

\section{LEGAL NOTICQ}

This repert was preparad as an eccount of Govarnment secessred watk.

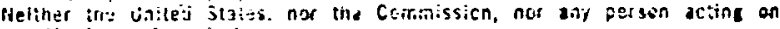
lenaif of ine iviniosion:

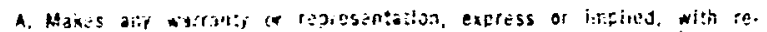

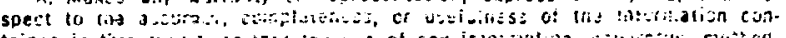

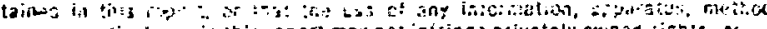

or process disciosed in this refert may nut initinge privately cwned $i$ :Gh:s: or

8. Acsurees anv liablilties with ressect to the use of or for daraang rasult. Ing trerz the use of any informatlon, aparaius, method, or precess disclosed in this report.

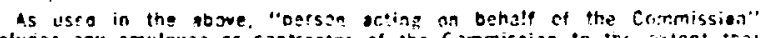

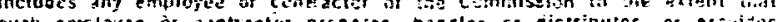
access to aty informotion ourstent to his emaloyment or contrait with

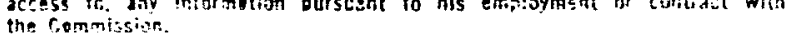

Submitted to Il Nuovo Cimento April, 1963

Contract No. AT(11-1)-264

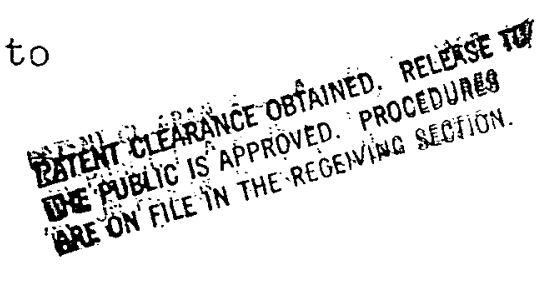

AUG 161963 


\title{
SELF-CONSISTENT MODELS OF STRONG INTERACTION WITH CHIRAL SYMMETRY*
}

\author{
Yoichiro Nambu \\ and \\ Pedro Pascual ${ }^{*}$
}

The Enrico Fermi Institute for Nuclear Studies and the Department of Physics

The University of Chicago, Chicago, Illinois

\begin{abstract}
We examine some simple models of (renormalizable) meson-nucleon interaction in which the nucleon mass is entirely due to interaction, and the chiral $\left(\gamma_{5}\right)$ symmetry is "broken" to become a hidden symmetry. We find that such a scheme is possible provided that a vector meson is introduced as an elementary field.
\end{abstract}

i

*Work supported by the U. S. Atomic Energy Commission. ** Present address: Junta de Energia Nuclear, Madrid, Spain. 
I. Introduction

In this paper we shall study some simple models of strong interactions in order to see whether the following points of view can be consistently entertained:

2) The mass of the fermion ("nucleon") arises entirely from boson-fermion interaction. In other words, the bare mass of the fermion field is zero.

b) The system may allow in adition the $\gamma_{5}$ invariance (chiral symmetry) which exhibits itselî as a "hidden symmetry," like in an earlier model (the superconductivity model) considered by Nambu and Jona-Lasinio ( NJ).

c) Some of the particles may in fact be non-elementary, composite systems in the sense of having vanishing renormalization constants.

By a hidden symmetry we mean a symmetry which is shared by the equations of motion of the system, but not by the states which make up the Hilbert space of the physical world. ${ }^{2}$ Such a possibility in the theory of elementary particles was demonstrated in NJ by taking analogy to the theory of superconductivity. This concept has also been exploited by Heisenberg, ${ }^{3}$ Goldstone, ${ }^{4}$ Marshak and Okubo, 5 Glashow, 6 etc.

In order to realize a hidden symmetry, it is necessary that the vacuum (ground) state of the world be not invariant under the symmetry operation considered, which means that the vacuum is not unique but degenerate. Each vacuum state will build upon it a set 
of excited states corresponding to the presence of particles, and the different "worlds" created by different vacuum states are orthogonal to each other. We regard the property of the vacuum state not as something given a priori in addition to the equations of motion, but as something that must follow from the latter dynamically.

We shall restrict ourselves in this paper to simple renormalizable models, in contrast to $\mathrm{NJ}$ and the Heisenberg theory where non-linear four-fermion interactions are introduced. Since we are interested in the bare masses and the bare couplings as well as the observed quantities, we introduce a cut off parameter to make the self-energies and renormalization constants finite.

As is known in the Lee model ${ }^{7}$ and various other model theories, the strength of the physical coupling constants cannot be chosen arbitrarily large in order to have a physically acceptable solution. 8 This condition expresses itself in the requirement that the amplitude renormalization constants $\left(z_{2}\right.$ and $\left.z_{3}\right)$ be within the range $0 \leq Z \leq 1$. Furthermore, we may regard the limiting case $z_{3}$ (or $z_{2}$ ) $=0$ as corresponding to a composite particle, 9 the meaning of which is not completely clear, but roughly indicates that the state of that particle can be constructed solely in terms of other fields.

In the following sections we consider models having either simple $\gamma_{5}$ symmetry or isotopic $\gamma_{5}$ symmetry. Neutral scalar, 
neutral and isovector pseudoscalar, and neutral vector fields are introduced in certain combinations to satisfy the symmetry properties. The calculation is carried out to the lowest order in the coupling constants and in the limit of large cut-off. The main conclusion is summarized in the last section.

II. Two-meson Model

Contrary to NJ, let us first assume a neutral nucleon, a pseudoscalar and scalar meson field coupled in a $\gamma_{5}$-invariant similar to the " $\sigma$ meson" model. 10
The Lagrangian is characterized by the bare nucleon mass $\mathrm{M}_{0}=a$, the bare meson masses $m_{\mathrm{so}_{0}}=\mathrm{m}_{\mathrm{po}}=\mathrm{m}_{\mathrm{o}}$, and the bare couplings $G_{s o}=G_{p o}=G_{0}$. The solution we seek is the one in which emerge a massive nucleon, a massive scalar meson and a massless pseudoscalar meson, the last being a simplified analog of the real pion (See, however, the remark at the end of Section IV.). The solution will be characterized by the observed masses $M \neq 0$, $\mathrm{m}_{\mathrm{s}} \neq \mathrm{m}_{\mathrm{p}}$, and the couplings $\mathrm{G}_{\mathrm{s}}^{2} \neq \mathrm{G}_{\mathrm{p}}^{2}$.

The general procedure is as follows. In NJ it was assumed that the real masses and coupling constants were expressed in terms of the bare constants of the theory as solutions of the self-consistent equations. In the present approach we shall express the bare masses and coupling constants in terms of the observed quantities. An elegant way to do this is to make use of the Lehmann-type formulas. 11,12 The symmetry properties reflected in the bare constants then impose conditions among the observed 
quantities. Namely, we write down the conditions

$$
\begin{aligned}
\mathrm{M}_{\mathrm{O}} & =0 \\
\mathrm{G}_{\mathrm{so}}^{2} & =\mathrm{G}_{\mathrm{po}}^{2} \\
\mathrm{~m}_{\mathrm{so}}^{2} & =m_{\mathrm{po}}^{2}
\end{aligned}
$$

in terms of renormalized quantities. (As will be seen below, the last equation is not crucial to our discussion).

In order to make the expressions inite, however, we have to introduce a cut-off mass parameter $\wedge$, and this spoils the elegance of the whole procedure. (This would also be necessary on dimensional grounas since otherwise we would have no mass scale). We shall therefore content ourselves with those cases where $\Lambda$ is large compared with $\mathrm{M}, \mathrm{m}_{\mathrm{s}}$ and thus the qualitative conclusions are insensitive to $\bigwedge$. In this case, the elaborate Lehmann type formulas will be unnecessary

We introduce, in the standard manner, ${ }^{13}$ the renormalization constants $z_{2}, z_{3 s}, z_{3 p}, z_{1 s}$ and $z_{1 p}$, and the self-energy integrals which we denote by $A$. Then

$$
\begin{aligned}
G_{s}^{2} & =Z_{l s}^{-2} \quad Z_{2}^{2} \quad Z_{3 s} G_{0}^{2}, \\
G_{p}^{2} & =z_{1 p}^{-2} \quad Z_{2}^{2} \quad Z_{3 p} G_{0}^{2},
\end{aligned}
$$

$$
\begin{aligned}
& \delta M=M-M_{0}=M=A_{2} / Z_{2}, \\
& \delta m_{S}^{2}=m_{S}^{2}-m_{0}^{2}=A_{3 s} / Z_{3 s}, \\
& \delta m_{p}^{2}=m_{p}^{2}-m_{0}^{2}=A_{3 p} / Z_{3 p} .
\end{aligned}
$$


For derivation of these formulas, see Ref. 13. By straightforward ... calculation, we find

$$
\begin{aligned}
& Z_{2}=1-s-p, \\
& Z_{3 s}=1-4 s, \quad Z_{3 p}=1-4 p \\
& Z_{1 s}=1-25+2 p, Z_{1 p}=1+2 s-2 p \\
& A_{2}=M(p-3 s) \\
& A_{3 s}=-\left(G_{s}^{2} / 8 \pi^{2}\right) \Lambda^{2}, A_{3 p}=-\left(G_{p}^{2} / 8 \pi^{2}\right) \Lambda^{2} \\
& s=\left(G_{s}^{2} / 32 \pi^{2}\right) \ln \left(\wedge^{2} / M^{2}\right), \quad p=\left(G_{p}^{2} / 32 \pi^{2}\right) \ln \left(\Lambda^{2} / M^{2}\right) .
\end{aligned}
$$

We have kept only the leading divergent terms in each of the expressions corresponding to the lowest order self-energy diagrams. ${ }^{14}$ we have ignored here the meson-meson interaction of the form $\lambda_{0}\left(\phi_{s}^{2}+\phi_{p}^{2}\right)^{2}$, which will only contribute additional terms to Eqs. (2.3b) ana (2.3c).

We also note that the formula for $\delta_{M}$ is valio only if $\left\langle\phi_{\mathrm{s}}(\mathrm{x})\right\rangle=0$, and $\left\langle\phi_{\mathrm{p}}(\mathrm{x})\right\rangle=0$. The latter is of course a consequence of parity conservation which we assume for the solution, but the former condition is not a priori dictated by our assumptions. In fact, $\left\langle\phi_{\mathrm{S}}\right\rangle \neq 0$ leads to a result similar to NJ, and this may be the simplest way of obtaining a non-trivial solution. In this paper, however, we will adopt the convention $\left\langle\phi_{s}\right\rangle=0$. The reason is that, as is discussed in the appendix, the contribution to the fermion self-energy coming from this effect becomes negligible in the limit of large cut-off.

A physically acceptable solution must satisfy 


$$
1 \geqslant z_{2} \geqslant 0, \quad 1 \geqslant z_{3 s} \geqslant 0, \quad 1 \geqslant z_{3 p} \geqslant 0
$$

From the last two, and $\mathrm{Eq} \cdot(2.4)$ we get

$$
s \leqslant 1 / 4, \quad p \leqslant 1 / 4 .
$$

The first condition is then autornatically guaranteed:

$$
z_{2} \geqslant 1 / 2
$$

However, the right-hand side of Eq. (2.3a) for the fermion mass now becomes 15

$$
M(p-3 s) /[1-(p+s)] \leqslant(1 / 3) M
$$

so that this equation cannot be satisfied. We conclude that our system does not have a self-consistent solution with $M \neq 0$. The interpretation of this result is that, as is clear from above, the scalar and pseudoscalar mesons do not produce a sufficient amount of fermion self-energy. This follows only from the requirement that the fermion mass be entirely due to self-energy, and does not depend on the condition $G_{\text {so }}^{2}=G_{p o}^{2}$. The same conclusion is reached when we consider a model which incorporates the isospin (see section IV.).

III. Vector Meson Model

Since spin $O$ mesons are not sufîicient to produce a self-consistent nucleon mass, we shall next add a neutral vector meson field to our picture. Its bare mass and bare coupling will be $m_{v o}$ and $G_{v o}$, and Eqs. (2.2) and (2.3) will be generalized in 
an obvious manner to include the vector meson. We now have

$$
\begin{aligned}
& \mathrm{z}_{2}=1-s-p-2 \mathrm{v} \\
& \mathrm{Z}_{3 \mathrm{~s}}=1-4 \mathrm{~s} \\
& \mathrm{Z}_{3 \mathrm{p}}=1-4 \mathrm{p} \\
& \mathrm{z}_{3 \mathrm{v}}=1-16 / 3 \mathrm{v} \\
& \mathrm{Z}_{1 \mathrm{~s}}=1+2 \mathrm{~s}-2 \mathrm{p}-8 \mathrm{v} \\
& \mathrm{Z}_{1 \mathrm{p}}=1-2 \mathrm{~s}+2 \mathrm{p}-8 \mathrm{v} \\
& \mathrm{Z}_{1 \mathrm{v}}=1-\mathrm{s}-\mathrm{p}-2 \mathrm{v}=\mathrm{Z}_{2} \\
& \mathrm{~A}_{2}=\mathrm{M}(-3 \mathrm{~s}+1 \mathrm{p}+6 \mathrm{v}) \\
& \mathrm{A}_{3 \mathrm{~s}}=-\frac{\mathrm{G}_{\mathrm{s}}{ }^{2}}{8 \pi^{2}} \Lambda^{2}
\end{aligned}
$$$$
A_{3 p}=-\frac{G_{p}^{2}}{8 \pi^{2}} \Lambda^{2}
$$$$
A_{3 v}=-\frac{G v^{2}}{16 \pi^{2}} \Lambda^{2}
$$$$
s=\frac{G_{s}^{2}}{32 \pi^{2}} \ln \frac{\Lambda^{2}}{M^{2}}, p=\frac{G_{p}^{2}}{32 \pi^{2}} \ln \frac{\Lambda^{2}}{M^{2}}
$$$$
v=\frac{G v^{2}}{32 \pi^{2}} \ln \frac{\Lambda^{2}}{M^{2}}
$$

From the condition $z_{3} \geqslant 0$, we get

$$
s \leqslant 1 / 4, \quad p \leqslant 1 / 4, v \leqslant 3 / 16,
$$

but in addition $z_{2} \geqslant 0$ requires

$$
s+p+2 v \leqslant 1 .
$$

The fermion self-energy relation becomes 


$$
\begin{aligned}
& M=M(-3 s+p+6 v) /(1-s-p-2 v) \\
& -s+p+4 v=1 / 2
\end{aligned}
$$

or

which is now consistent with Eqs. (3.2) and (3.3) if

$$
\mathrm{v}=1 / 8+\frac{\mathrm{s}-\mathrm{p}}{4} \geqslant 1 / 16
$$

Further we see that Eq. (3.4) means

$$
z_{1 s}=0, z_{1 p}=4(p-s)
$$

Next consider the condition (2.1b) for the equality of $G_{S O}^{2}$ and $G_{\mathrm{po}}^{2} \cdot$ Eqs. $(2.2)$ and $(2.3)$ give

$$
p(1-2 s+2 p-8 v)^{2}(1-4 s)=s(1-4 p)(1+2 s-2 p-8 v)^{2}
$$

which leads to

a) $s-p=0$

or

b) $(1-8 v)^{2}+4(p-s)^{2}-4(1-8 v)(8 p s-s-v)=0 \quad(3.7)$

Combining Eqs. (3.4) and (3.7), we obtain three solutions

a)

$$
\begin{aligned}
& \mathrm{s}=\mathrm{p}<1 / 4, \quad \mathrm{v}=1 / 8 \\
& z_{1 S}=z_{1 p}=0, z_{3 s}=z_{3 p} \text {, } \\
& \text { b) } \quad s=1 / 4, \quad z_{3 s}=z_{1 s}=0 \\
& \text { c) } \quad p=0, z_{3 p}=1, z_{1 p} \leqslant 0 \text {. }
\end{aligned}
$$

The solution $b)$, however, corresponds to an indefinite form $G_{S .0}^{2} / G_{p o}^{2}=0 / 0$, so that we will replace it by a stronger one

$$
\begin{aligned}
& \mathrm{b} \text { “) } \quad \mathrm{p}=\mathrm{s}=1 / 4, \quad \mathrm{v}=1 / 8, \\
& \mathrm{z}_{1 \mathrm{p}}=\mathrm{z}_{1 \mathrm{~s}}=\mathrm{z}_{3 \mathrm{p}}=\mathrm{z}_{3 \mathrm{~s}}=0, \mathrm{z}_{2}=1 / 4,
\end{aligned}
$$

which is a limiting case of a). 
The relation $z_{1}=0$ means that the bare coupling is zero, whereas $\mathrm{z}_{3}=0$ means that there is no free energy term for the bare field in the Lagrangian. The latter is often considered to be characteristic of composite particles. 9 In the case b') above, then, the scalar and pseudoscalar mesons are bound states induced by the vector field (which causes attraction between fermion pairs).

In the case $c$ ), the pseudoscalar coupling constant is zero, which means that the pseudoscalar field is dynamically decoupled at least within our approximation from the rest of the system. It is rather remarkable that one can still talk about $\gamma_{5}$ symmetry, but the significance of such a solution is not clear.

Finally, the third condition (3.1c), together with Eqs. (2.3b) and (2.3c) means

$$
m_{s}^{2}-m_{p}^{2}=A_{3 s} / Z_{3 s}-A_{3 p} / Z_{3 p} \cdot
$$

This equation, however, does not amount to a self-consistency requirement in our approximation where the right-hand side is independent of $m_{S}$ and $m_{p}$. Our solutionsa) and $b^{\prime}$ ) clearly mean $m_{s}^{2}=m_{p}^{2}$, and $m_{o}^{2}$ itself will tend to $\infty$ as $\mathrm{z}_{3 \mathrm{~s}}, \mathrm{z}_{3 \mathrm{p}} \rightarrow 0$.

The equality of the scälar and pseudoscalar meson masses in these cases a) and $b^{\prime}$ ) are only due to our approximation of keeping the leading divergent terms. If we take into account the correction terms, all the expressions in Eq. (3.1) 
will have to be modified, and so will the solutions (3.8) and (3.81). The first order mass split $m_{s}{ }^{2}-m_{p}{ }^{2}=\Delta$ may be obtained by putting $m_{s}=m_{p}$, and $s=p$ in computing the $Z$ 's and the A's. We find

$$
\begin{aligned}
& A_{3 s}-A_{3 p}=16 \Delta M^{2} \\
& z_{3 s}-z_{3 p}=0\left(\Lambda^{0}\right)>0
\end{aligned}
$$

Neglecting the difference of the $z_{3}$ 's since it is convergent, we get (for the cases a) ana $\left.b^{\prime}\right)$ )

$$
m_{s}^{2}-m_{p}^{2}=16 \Delta \mathrm{m}^{2} / z_{3}>0
$$

It has often been asserted ${ }^{1,4}$ that the breaking of $\gamma_{5}$ invariance in our fashion should necessarily lead to $m_{p}=0$. This, however, is a rather subtle property which does not follow from our formulas alone. The results of the present paper are independent of this question.

IV. Vector Meson Model with Isospin

We consider here a simple generalization of the preceding model by assuming a fermion field of isospin 1/2, a scalar ( $s$ ), a pseudoscalar ( $p)$, and a vector field of isospin 0 , and a pseudoscalar ( $\left.p^{\prime}\right)$ field of isospin 1 . If $G_{\mathrm{so}}=G_{p o}$, we have a simple $\gamma_{5}$ invariance, and if $G_{S_{0}}=G_{p^{\prime} 0}$, we have an isotopic $\gamma_{5}$ invariance. The former turns out to have only one solution: $p=0$, corresponding to case $c$ ) of $\mathrm{Eq} \cdot(3.8)$, due to the fact that the condition $z_{3 v} \geqslant 0$ cannot be met in the other 
cases. So we shall consider in the following only the isotopic $\gamma_{5}$ invariance. Eq. (3.1) is now replaced by

$$
\begin{aligned}
& z_{2}=1-s-p-3 p^{\prime}-2 v \\
& z_{3 s}=1-8 s, z_{3 p}=1-8 p \\
& z_{3 p^{\prime}}=1-8 p^{\prime}, z_{3 v}=1-\frac{32}{3} v \\
& z_{1 s}=1+2 s-2 p-6 p^{\prime}-8 v \\
& z_{1 p}=1-2 s+2 p+6 p^{\prime}-8 v \\
& z_{1 p^{\prime}}=1-2 s+2 p-2 p^{\prime}-8 v \\
& z_{1 v}=z_{2} \\
& A_{2}=(-3 s+p+6 v) M
\end{aligned}
$$$$
A_{3 s}=-\frac{G_{s}^{2}}{4 \pi^{2}} \Lambda^{2}, \quad A_{3 p}=-\frac{G_{p}^{2}}{4 \pi^{2}} \Lambda^{2}
$$

$$
A_{3 p^{\prime}}=-\frac{G_{p^{\prime}}{ }^{2}}{4 \pi^{2}} \Lambda^{2}, \quad A_{3 v}=-\frac{G_{v}{ }^{2}}{8 \pi^{2}} \Lambda^{2}
$$

Eqs. (3.2) and (3.3) are replaced by

$$
\mathrm{s} \leqslant 1 / 8, \quad \mathrm{p} \leqslant 1 / 8, \quad \mathrm{p} ! \leqslant 1 / 8, \quad \mathrm{v} \leqslant 3 / 32 \quad(4.2)
$$

and

$$
s+p+3 p^{\prime}+2 v \leqslant 1
$$

respectively.. The two crucial relations (3.4) and (3.7) are replaced by

$$
\begin{array}{r}
-2 s+2 p+6 p^{\prime}+8 v=1, \\
p^{\prime}\left(-s+p+p^{\prime}\right)^{2}(1-8 s)=0 . x \cdot s\left(1-8 p^{\prime}\right)=0
\end{array}
$$

respectively, since the $Z^{\prime}$ s reduce simply to 


$$
\begin{aligned}
& z_{1 s}=0 \\
& z_{1 p}=-4 s+4 p+12 p^{\prime} \\
& z_{1 p^{\prime}}=-4 s+4 p+4 p^{\prime}
\end{aligned}
$$

Eqs. (4.4a) and (4.4b) express two conditions among the four constants $s, p, p^{\prime}$ and $v$.

Proceeding as before, we get three solutions
a) $\mathrm{s}=\mathrm{p}+\mathrm{p}^{\prime}$,
$z_{I S}=z_{I p^{\prime}}=0$
b) $\mathrm{s}=\mathrm{p}^{\prime}=1 / 8$,
$\mathrm{p}=0, \quad \mathrm{v}=1 / 16$
c) $p^{\prime}=0, z_{3 p^{\prime}}=1, z_{1 s}=0$.

V. Conclusion

The moral of this admittedly crude study seems to be the following:

a) The assumption that the fermion mass arises entirely from interaction with boson fields is not always selfconsistent. This assumption is only part of the $\gamma_{5}$ invariance requirement and may be imposed on its own merit from a different point of view. In particular, spin o fields are not sufficient to build up the fermion mass, but a vector field will do.

b) Once the first condition is satisfied, it is also possible to impose the full $\gamma_{5}$ invariance condition which introduces further relations among the coupling constants.

c) With the presence of a vector field, it is possible 
to consider the scalar and pseudoscalar mesons as composite in the sense that $z_{3}=0$. But the vector meson itself and the fermion seem to remain elementary in the models we have studied.

We point out, incidentally, that if only the assumption a) is kept, one can make $z_{2}=0$. Because of the relation (2.3a), one must demand $A_{2}=0$ for consistency, so the condition a) is also necessary for $z_{2}=0$. Taking the model of Section 3 , we find a solution

$$
\begin{aligned}
& 1-2 s=1 / 2-2 v \\
& 1-2 p=-1 / 2+6 v
\end{aligned}
$$

One easily sees that $\mathrm{v}$ and $\mathrm{s}$ cannot be zero, and that all the mesons are elementary. It is interesting, perhaps, to compare this result with the conjecture ${ }^{16}$ regarding the "compositeness" of a fermion, in which a vector field was found necessary (but may not be sufficient) to make the fermion a possible Regge pole. We thank Dr. D. Lurie for useful discussions. 
$-15-$

Appendix

We shall study here the possibility of assuming $\left\langle\phi_{S}(x)\right\rangle \neq 0$. If this is the case, we have an extra contrabution, to be called $\mathrm{M}_{2}$, to the fermion self-energy in addion to the ordinary type called $\mathrm{M}_{1}$. Thus

$$
\begin{aligned}
\mathrm{M}_{2} & =\mathrm{G}_{\mathrm{SO}}\left\langle\phi_{\mathrm{S}}(\mathrm{x})\right\rangle \\
& =-\left(\mathrm{G}_{\mathrm{SO}}^{2} / \mathrm{m}_{\mathrm{SO}}^{2}\right) \mathrm{T}_{\mathrm{r}} \mathrm{S}_{\mathrm{u}}(0) \\
& =-\left(\mathrm{G}_{\mathrm{SO}}^{2} / \mathrm{m}_{\mathrm{SO}}^{2}\right) \mathrm{Z}_{2} \mathrm{~T}_{\mathrm{r}} \cdot \mathrm{S}(0)
\end{aligned}
$$

The second line follows from the equation of motion for $\phi_{\mathrm{S}}$ :

$$
\left(\square-m_{s 0}^{2}\right) \varnothing_{S}=G_{S O} \bar{\psi} \psi
$$

All the fields here are unrenomalized, and so is the fermion propagator $S_{u}(x)$.

Now the renormalized $S(x)$ is expressed in the Lehmann representation of the type

$$
\begin{aligned}
S(x)= & \gamma_{\mu} \partial / \partial x_{\mu} \int_{0}^{\infty} \Delta^{(x)}(x) \rho_{1}(x) d x \\
& -\int_{0}^{\infty} \Delta^{(x)}(x) \rho_{2}(x) d x
\end{aligned}
$$

where $\Delta^{(\mathscr{L})}$ is the Feynman propagator with mass $\mathscr{H}$. Then one can show that

$$
\begin{gathered}
Z_{2}^{-1}=\int_{0}^{\infty} \rho_{1} d x \\
\left(M_{0}+M_{2}\right) \int_{0}^{\infty} \rho_{1} d x=\int_{0}^{\infty} \rho_{2} d x
\end{gathered}
$$


following Lehmann's derivation, but paying due attention to the fact that $\langle\phi\rangle \neq 0$.

$$
\begin{aligned}
& \text { We have thus } \\
& \begin{aligned}
\operatorname{Tr} S(0) & =-4 \int_{0}^{\infty} \Delta^{(x)}(0) \rho_{2} d x \\
& =-4 \Delta^{(0)}(0) \int \rho_{2} d x+R \\
& =4 \Delta^{(0)}(0) Z_{2}^{-1}\left(M_{0}+M_{2}\right)+R
\end{aligned}
\end{aligned}
$$

where $\Delta^{(0)}(0)=\left[-i /(2 \pi)^{4}\right] \int \mathrm{d}^{4} \mathrm{p} /\left(\mathrm{p}^{2}-i \varepsilon\right)$ is quadratically divergent, whereas the remainder $R$ is at most logarithmically divergent. Eq. (A.I) becomes, setting $M_{0}=0$,

$$
M_{2}=4 G_{50}^{2} \Delta^{(0)}(0) M_{2} / m_{s_{0}}^{2}+G_{50}^{2} Z_{2} R / m_{s_{0}}^{2}
$$

We note that both $\Delta^{(0)}(0)$ and $m_{\text {so }}^{2}$ are quadratically divergent, so that $G_{\text {SO }}^{2} \Delta^{(0)}(0) / m_{\text {SO }}^{2}=O(1)$. Then $M_{2} / M$ is of the order of $\left(\mathbb{M}^{2} / \Lambda^{2}\right) \ln \left(\Lambda^{2} / \mathbb{M}^{2}\right) \sim 0$ in our high cut-off limit. Thus it is consistent to assume $\left\langle\phi_{\mathrm{s}}\right\rangle=0$, and the bulk of the fermion self-energy comes from the ordinary type $M_{1}$. 
$\underline{\text { References }}$

1. Y. Nambu and G. Jona-Lasinio, Phys. Rev. 122, 345 (1961)

2. Y. Nambu, Lectures at The NATO Advanced Study Institute in Istanbul, July 1962 .

3. See, for example, H.P. Durr and W. Heisenberg, Zeit. f. Naturforschung, $16 \mathrm{a}, 726$ (1961).

4. J. Goldstone, Nuovo Cimento 19, 154 (1961).

J. Goldstone, A. Salam and S. Weinberg, to be published.

5. R. E. Marshak and S: Okubo, Nuovo Cimento 19, 1226 (1961).

6. S. Glashow, to be published.

7. T. D. Lee, Phys. Rev. 95, 1329 (1956); G. Källén and W. Pauli, Kgl. Danske Vidensk. Selsk. Mat-Fys. Medd 29, No. 17 (1955).

8. H. Ruderman and S. Gasiorowicz, Nuovo Cimento 8, 861 (1958); V. N. Gribov, Ya. B. Zel'dovitch and A. M. Perelomov, J. Exptl. Theoret. Phys. 40, 1190 (1961).

9. J. C. Howard and B. Jouvet, Nuovo Cimento 18, 466 (1960);

M. J. Vaughn, R. Aaron, and R. D. Amado, Phys. Rev. 124, 1258 (1961): A. Salam, Nuovo Cimento 25, 224 (1962);

J. S. Dowker, Nuovo Cimento 25, 1135 (1962); S. Weinberg to be published.

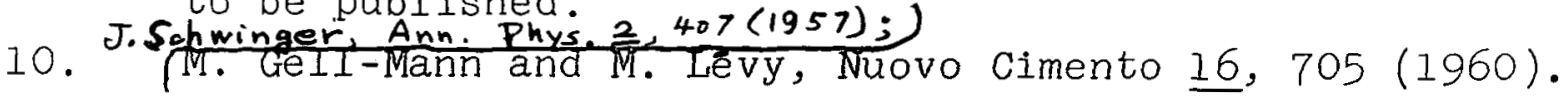

11. H. Lehmann, Nuovo Cimento 11, 342 (1956); E. Ferrari and G. Jona-Lasinio, Nuovo Cimento 10, 310 (1958), 16, 867 (1960).

12. H. Lehmann, K. Symanzik and W. Zimmermann, Nuovo Cimento 2. 425 (1955); K. Ford, Phys. Rev. 105, 320 (1956). 13. F. J. Dyson, Phys. Rev. 75, 1736 (1949); P. T. Matthews and A. Salam, Phys. Rev. 94, 185 (1954). Eqs. (2.3) and (2.4) do not conform to the original Lehmann formula, ${ }^{11}$ but rather to the alternative formula, 12 in which the inverse of a propagator is expressed by a spectral 
representation. This has an advantage in imposing the physical condition on $Z$.

14. The cut-off is here done by casting the divergent expressions into spectral forms with upper limit $\wedge$. For logarithmic divergences, the result is independent of the way of cutting off to within convergent terms.

15. We are deviating from a strict power series expansion by keeping the factor $z_{2}$. Dropping $z_{2}$ would change some of the quantitative results below.

16. M. Gell-Mann and M. L. Goldberger, Phys. Rev. Lett. q, 275 (1962). 